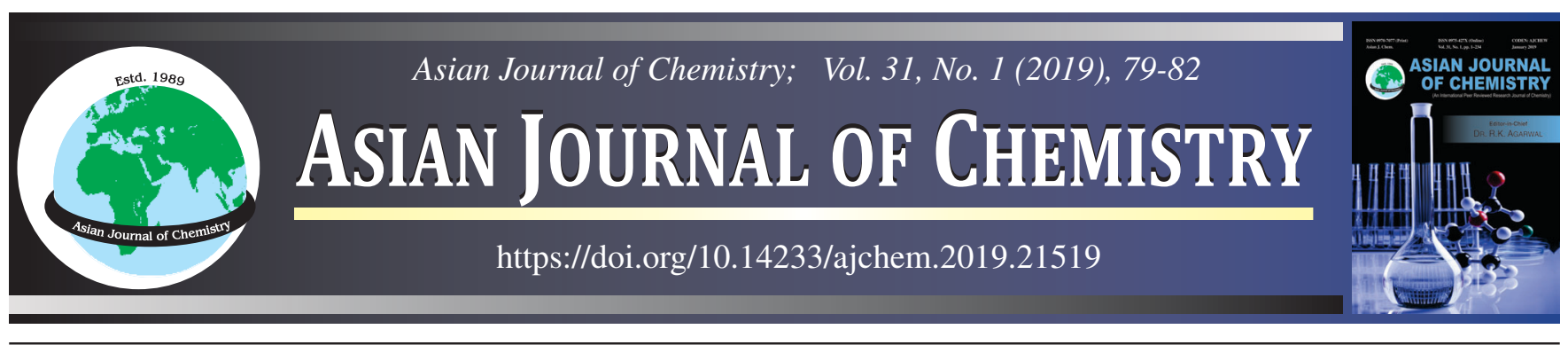

\title{
Synthesis and Antimicrobial Activities of Iminothioether Linkers on Cyclopenta[a]phenanthrene Derivatives
}

\author{
Balasaheb P. Pagar
}

Post Graduate Department of Chemistry, K.S.K.W. Arts, Science \& Commerce College, Uttamnagar, CIDCO, Nashik-422008, India

Corresponding author: E-mail: pagarbp77@gmail.com

Received: 15 June 2018;

Accepted: 14 August 2018;

Published online: 30 November 2018;

AJC-19162

The reaction of methylbenzo[h][1,6]naphthyridine $(\mathbf{1})$ with thiourea yield 4-chloro-12-methyl-16,17-dihydro-15-thia-6,11-diazacyclopenta[ $a]$ phenanthrene-7-thiol (2). The chemistry of compound $\mathbf{2}$ is explored to obtained iminothioether derivatives (3a-h) in good yields. The structures of newly synthesized compounds were confirmed by spectral data and elemental analysis. The antimicrobial activity of newly synthesized compounds were studied against Staphylococcus aureus, Escherichia coli, Bacillus subtilis, Pseudomonas aeroginosa, Proteus valgaris, Bacillus cereus, Streptococcus sp. and Bacillus megaterium by the agar well diffusion method. Compounds $\mathbf{3 d ,} \mathbf{3 f}$ and 3h showed moderate antimicrobial activity.

Keywords: Benzo $[h][1,6]$ naphthyridine, cyclopenta $[a]$ phenanthrene, Antimicrobial.

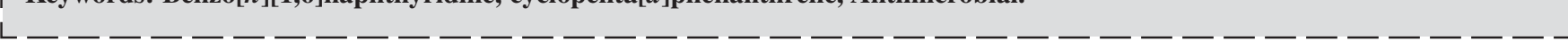

\section{INTRODUCTION}

Benzo[1,6]naphthyridine derivatives are class of fused heterocyclic compounds that exhibit broad spectrum of biological activities such as antimalarial [1], inhibitor of HIV-1 integrase [2-5], HCMV [6,7], FGF receptor-1 tyrosine kinase [8] and the enzyme acetyl cholinesterase [9,10]. The SAR studies of these types of compounds were having quinoline nucleus as carrier of several cytotoxic agents [11,12]. Recent discovery of benzo[1,6]naphthyridine as selective highly potent mammalian inhibitor of rapamycin (mTOR) for the treatment of cancer cell $[13,14]$. In view of all these reports we have synthesized bioactive naphthyridine heterocyclic derivatives using $\alpha$-acetyl $\gamma$-butyrolactone and heterocyclic amines, [15-21] to investigate new derivatives of benzo[ $h][1,6]$ naphthyridines. In this present communication we report the synthesis of $\mathrm{C}_{7^{-}}$ iminotiaoether linkers to cyclopenta[ $a]$ phenanthren-7-ylsulfanyl)- $N$-phenyl acetamide.

\section{EXPERIMENTAL}

Commercially available common reagents was used for synthesis or synthesized by standard literature known methods. Open capillary tube method was used to measure melting points on Barnstead Electro Thermal apparatus Mod. No. IA-
9200. The thermo Quest flash EA 1112-Elemental Analyzer was used for elemental analysis of synthesized compounds. The IR spectra were recorded on Shimadzu IR 408 instrument and the mass spectra recorded on Varian Mat Bremen mass spectrometer Mat 112. The ${ }^{1} \mathrm{H}$ NMR spectra (300 MHz) and ${ }^{13} \mathrm{C}$ NMR $\left(75 \mathrm{MHz}\right.$ ) were tun on VARIAN XL-300 in $\mathrm{CDCl}_{3}$ or DMSO- $d_{6}$ using TMS as an internal standard. Chemical shift $(\delta)$ are measured in ppm, coupling constants $(J)$ in $\mathrm{Hz}$. All reactions were checked by using TLC $0.2 \mathrm{~mm}$ silica gel $60 \mathrm{~F}_{254}$ plates using UV light for detection 254 and $366 \mathrm{~nm}$.

\section{Synthetic procedures}

4-Chloro-12-methyl-16,17-dihydro-15-thia-6,11-diazacyclopenta $[a]$ phenanthrene-7-thiol (2): A mixture of compound 1 (3.60 g, $0.01 \mathrm{~mol})$ and thiourea $(0.98 \mathrm{~g}, 0.013$ $\mathrm{mol})$ in acetic acid $(20 \mathrm{~mL})$ was refluxed for $20 \mathrm{~min}$. The reaction completion was monitored by using TLC (toluene/ ethyl acetate 8:2) and reaction mixture was slowly poured in $300 \mathrm{~mL}$ stirred ice cold water. The crude product was isolated by suction filtration and recrystallized by using ethanol and DMF gave 4-chloro-12-methyl-16,17-dihydro-15-thia-6,11diazacyclopenta[ $a]$ phenanthrene-7-thiol (2); Yield (2.86 g, 90 $\%$ ); m.p.: $242{ }^{\circ} \mathrm{C}$; IR (KBr, $\left.v_{\max }, \mathrm{cm}^{-1}\right): 3350(\mathrm{SH}), 3330(\mathrm{NH})$, 2923, 2852, $1660(\mathrm{C}=\mathrm{S}), 1543,1432,1214,766 ;{ }^{1} \mathrm{H}$ NMR in

This is an open access journal, and articles are distributed under the terms of the Creative Commons Attribution-NonCommercial 4.0 International (CC BY-NC 4.0) License, which allows others to copy and redistribute the material in any medium or format, remix, transform, and build upon the material, as long as appropriate credit is given and the new creations are licensed under the identical terms. 
$\mathrm{CDCl}_{3}: \delta 2.61\left(\mathrm{~s}, 3 \mathrm{H}, \mathrm{CH}_{3}\right), 3.34\left(\mathrm{t}, J=7.0 \mathrm{~Hz}, 2 \mathrm{H}, \mathrm{CH}_{2} \mathrm{CH}_{2} \mathrm{~S}\right)$, $3.38\left(\mathrm{t}, J=7.0 \mathrm{~Hz}, 2 \mathrm{H}, \mathrm{CH}_{2} \mathrm{CH}_{2} \mathrm{~S}\right), 7.38(\mathrm{t}, J=6.9 \mathrm{~Hz}, 1 \mathrm{H}$, $\left.\mathrm{C}_{2} \mathrm{H}\right), 7.59\left(\mathrm{~d}, J=6.9 \mathrm{~Hz}, 1 \mathrm{H}, \mathrm{C}_{3} \mathrm{H}\right), 8.73(\mathrm{~d}, J=6.9 \mathrm{~Hz}, 1 \mathrm{H}$, $\left.\mathrm{C}_{1} \mathrm{H}\right), 10.41$ (s, 1H, SH); MS: $m / z$ (\%): $320(\mathrm{M}+2,40), 318$ (M, 100), 283 (30), 248 (20), 159 (10); Anal. Calcd. for $\mathrm{C}_{15} \mathrm{H}_{11} \mathrm{~N}_{2} \mathrm{~S}_{2} \mathrm{Cl}$ (318.85): C, 56.51; H, 3.48; N, 8.79. Found: C, 56.42; H, 3.37; N, 8.74.

(4-Chloro-12-methyl-16,17-dihydro-15-thia-6,11-diazacyclopenta $[a]$ phenanthren-7-ylsulfanyl)acetic acid ethyl ester (3a): Anhydrous potassium carbonate ( $2.04 \mathrm{~g}, 0.015 \mathrm{~mol})$ was added to the stirred solution of 4-chloro-12-methyl-16,17dihydro-15-thia-6,11-diazacyclopenta[ $a$ ] phenanthrene-7thiol (2) (4.77 g, $0.015 \mathrm{~mol})$ and ethyl bromoacetate $(3.6 \mathrm{~mL}$, $0.018 \mathrm{~mol})$ in DMF $(50 \mathrm{~mL})$ at $25^{\circ} \mathrm{C}$. Stirring the resulting solution for $2 \mathrm{~h}$ and the progress of reaction was confirmed by using TLC. After completion, the resulting solution was slowly added in cold water $500 \mathrm{~mL}$. The precipitated solid was collected by suction filtration, washed with water and recrystallized in DMF/ethanol (2:8) to yield compound 3a; Yield $(5.51 \mathrm{~g}, 91$ \%); m.p.: $163{ }^{\circ} \mathrm{C}$; IR (KBr, $\left.v_{\max }, \mathrm{cm}^{-1}\right)$ : 2976, 2929, 1739 $(\mathrm{C}=\mathrm{O}), 1543,1296,1159,954 ;{ }^{1} \mathrm{H}$ NMR $\left(\mathrm{CDCl}_{3}\right): \delta 1.29(\mathrm{t}, J$ $\left.=7.2 \mathrm{~Hz}, 3 \mathrm{H}, \mathrm{OCH}_{2} \mathrm{CH}_{3}\right), 2.67\left(\mathrm{~s}, 3 \mathrm{H}, \mathrm{CH}_{3}\right), 3.45(\mathrm{t}, J=7.8$ $\mathrm{Hz}, 2 \mathrm{H}, \mathrm{CH}_{2} \mathrm{CH}_{2} \mathrm{~S}$ ), 3.56 (t, $J=7.8 \mathrm{~Hz}, 2 \mathrm{H}, \mathrm{CH}_{2} \mathrm{CH}_{2} \mathrm{~S}$ ), 4.25 (q, $\left.J=7.2 \mathrm{~Hz}, 2 \mathrm{H}, \mathrm{OCH}_{2} \mathrm{CH}_{3}\right), 4.29\left(\mathrm{~s}, 2 \mathrm{H}, \mathrm{CH}_{2}\right), 7.43(\mathrm{t}, J=$ $\left.8.1 \mathrm{~Hz}, 1 \mathrm{H}, \mathrm{C}_{2} \mathrm{H}\right), 7.74\left(\mathrm{~d}, J=8.1 \mathrm{~Hz}, 1 \mathrm{H}, \mathrm{C}_{3} \mathrm{H}\right), 8.90(\mathrm{~d}, J=$ $\left.8.1 \mathrm{~Hz}, 1 \mathrm{H}, \mathrm{C}_{1} \mathrm{H}\right)$; MS: m/z (\%): $407(\mathrm{M}+2,50), 405(\mathrm{M}+$, 100), 311 (20), 195 (30), 134 (70), 100 (10). Anal. Calcd. for $\mathrm{C}_{19} \mathrm{H}_{17} \mathrm{~N}_{2} \mathrm{O}_{2} \mathrm{~S}_{2} \mathrm{Cl}$ (404.94): C, 56.36; H, 4.23; N, 6.92. Found: C, 56.30; H, 4.20; N, 6.94.

2-(4-Chloro-12-methyl-16,17-dihydro-15-thia-6,11diazacyclopenta[ $[a]$ phenanthren-7-ylsulfanyl)-N-substituted phenyl acetamide (3b-h): Anhydrous potassium carbonate $(0.136 \mathrm{~g}, 0.001 \mathrm{~mol})$ was added to the stirred solution of 4-chloro12-methyl-16,17-dihydro-15-thia-6,11-diazacyclopenta[a]phenanthrene-7-thiol (2) $(0.318 \mathrm{~g}, 0.001 \mathrm{~mol})$ and 2-bromo$\mathrm{N}$-phenyl-acetamide $(0.0012 \mathrm{~mol})$ in DMF at $25^{\circ} \mathrm{C}$. Stirring the resulting solution for $2 \mathrm{~h}$ and the progress of reaction was confirmed by using TLC. After completion, the resulting solution was slowly added in cold water $(100 \mathrm{~mL})$ and the solid obtained was recrystallized in DMF/ethanol (2:8) to yield iminothioether derivatives (3b-h).

2-(4-Chloro-12-methyl-16,17-dihydro-15-thia-6,11diazacyclopenta $[a]$ phenanthren-7-ylsulfanyl)-N-p-tolylacetamide (3b): Yield $\left(0.396\right.$ g, $85 \%$ ); m.p.: $266^{\circ} \mathrm{C}$; IR (KBr, $\left.v_{\max }, \mathrm{cm}^{-1}\right): 3338(\mathrm{NH}), 2918,1685(\mathrm{C}=\mathrm{O}), 1600,1543,1440$, $1257,1166,1126,950 ;{ }^{1} \mathrm{H} \mathrm{NMR}\left(\mathrm{CDCl}_{3}\right): \delta 2.24\left(\mathrm{~s}, 3 \mathrm{H}, \mathrm{CH}_{3}\right)$, $2.70\left(\mathrm{~s}, 3 \mathrm{H}, \mathrm{CH}_{3}\right), 3.47\left(\mathrm{t}, J=7.2 \mathrm{~Hz}, 2 \mathrm{H}, \mathrm{CH}_{2} \mathrm{CH}_{2} \mathrm{~S}\right), 3.56(\mathrm{t}$, $\left.J=7.2 \mathrm{~Hz}, 2 \mathrm{H}, \mathrm{CH}_{2} \mathrm{CH}_{2} \mathrm{~S}\right), 4.30\left(\mathrm{~s}, 2 \mathrm{H}, \mathrm{CH}_{2}\right), 7.03$ (d, $J=7.8$ $\mathrm{Hz}, 2 \mathrm{H}, \mathrm{ArH}), 7.37(\mathrm{~d}, J=7.8 \mathrm{~Hz}, 2 \mathrm{H}, \mathrm{ArH}), 7.52(\mathrm{t}, J=7.8$ $\left.\mathrm{Hz}, 1 \mathrm{H}, \mathrm{C}_{2} \mathrm{H}\right), 7.85\left(\mathrm{~d}, J=7.8 \mathrm{~Hz}, 1 \mathrm{H}, \mathrm{C}_{3} \mathrm{H}\right), 7.98(\mathrm{~d}, J=7.8$ $\left.\mathrm{Hz}, 1 \mathrm{H}, \mathrm{C}_{1} \mathrm{H}\right), 9.77(\mathrm{~s}, 1 \mathrm{H}, \mathrm{NH}) ;{ }^{13} \mathrm{C}$ NMR $\left(\mathrm{CDCl}_{3}\right): \delta$; MS: $\mathrm{m} / z$ (\%): 468 (M+2, 20), 466 (M+, 31), 340 (50), 318 (100), 202 (50), 160 (40), 91 (30). Anal. Calcd. for $\mathrm{C}_{24} \mathrm{H}_{20} \mathrm{~N}_{3} \mathrm{OS}_{2} \mathrm{Cl}$ (466.03): C, 61.86; H, 4.33; N, 9.02. Found: C, 61.84; H, 4.30; $\mathrm{N}, 9.04$.

2-(4-Chloro-12-methyl-16,17-dihydro-15-thia-6,11diazacyclopenta $[a]$ phenanthren-7-ylsulfanyl)-N-(4-fluorophenyl)acetamide (3c): Yield $(0.408$ g, $87 \%)$; m.p.: $256{ }^{\circ} \mathrm{C}$;
IR $\left(\mathrm{KBr}, v_{\max }, \mathrm{cm}^{-1}\right): 3348(\mathrm{NH}), 2918,1685(\mathrm{C}=\mathrm{O}), 1545$, $1506,1404,1211,1165,950 ;{ }^{1} \mathrm{H}$ NMR $\left(\mathrm{CDCl}_{3}\right): \delta 2.71$ (s, $\left.3 \mathrm{H}, \mathrm{CH}_{3}\right), 3.46\left(\mathrm{t}, J=7.0 \mathrm{~Hz}, 2 \mathrm{H}, \mathrm{CH}_{2} \mathrm{CH}_{2} \mathrm{~S}\right), 3.56(\mathrm{t}, J=7.0$ $\left.\mathrm{Hz}, 2 \mathrm{H}, \mathrm{CH}_{2} \mathrm{CH}_{2} \mathrm{~S}\right), 4.40\left(\mathrm{~s}, 2 \mathrm{H}, \mathrm{CH}_{2}\right), 7.31(\mathrm{~d}, J=8.2 \mathrm{~Hz}, 2 \mathrm{H}$, $\mathrm{ArH}), 7.51\left(\mathrm{t}, J=7.6 \mathrm{~Hz}, 1 \mathrm{H}, \mathrm{C}_{2} \mathrm{H}\right), 7.67(\mathrm{~d}, J=8.2 \mathrm{~Hz}, 2 \mathrm{H}$, $\mathrm{ArH}), 7.83\left(\mathrm{~d}, J=7.6 \mathrm{~Hz}, 1 \mathrm{H}, \mathrm{C}_{3} \mathrm{H}\right), 8.91(\mathrm{~d}, J=7.6 \mathrm{~Hz}, 1 \mathrm{H}$, $\left.\mathrm{C}_{1} \mathrm{H}\right), 10.01$ (s, 1H, NH); MS: $\mathrm{m} / z$ (\%): $471(\mathrm{M}+2,20), 469$ (M+, 40), 418 (30), 361 (40), 319 (70), 151 (100), 91 (30). Anal. Calcd. for $\mathrm{C}_{23} \mathrm{H}_{17} \mathrm{FN}_{3} \mathrm{OS}_{2} \mathrm{Cl}(469.99)$ : C, 58.78; H, 3.65; N, 8.94. Found: C, 58.72; H, 3.64; N, 8.96.

2-(4-Chloro-12-methyl-16,17-dihydro-15-thia-6,11diazacyclopenta $[a]$ phenanthren-7-ylsulfanyl)-N-(4chloro-phenyl)acetamide (3d): Yield $(0.398 \mathrm{~g}, 82 \%)$; m.p.: $262{ }^{\circ} \mathrm{C}$; IR (KBr, $\left.v_{\max }, \mathrm{cm}^{-1}\right): 3355(\mathrm{NH}), 2926,2840,1683$ $(\mathrm{C}=\mathrm{O}), 1594,1510,1318,1224,850 ;{ }^{1} \mathrm{H}$ NMR $\left(\mathrm{CDCl}_{3}\right): \delta$ 2.72 (s, $\left.3 \mathrm{H}, \mathrm{CH}_{3}\right), 3.46$ (t, $\left.J=7.6 \mathrm{~Hz}, 2 \mathrm{H}, \mathrm{CH}_{2} \mathrm{CH}_{2} \mathrm{~S}\right), 3.57$ (t, $\left.J=7.6 \mathrm{~Hz}, 2 \mathrm{H}, \mathrm{CH}_{2} \mathrm{CH}_{2} \mathrm{~S}\right), 4.41\left(\mathrm{~s}, 2 \mathrm{H}, \mathrm{CH}_{2}\right), 7.35(\mathrm{~d}, J=8.3$ $\mathrm{Hz}, 2 \mathrm{H}, \mathrm{ArH}), 7.54\left(\mathrm{t}, J=7.8 \mathrm{~Hz}, 1 \mathrm{H}, \mathrm{C}_{2} \mathrm{H}\right), 7.61(\mathrm{~d}, J=8.3$ $\mathrm{Hz}, 2 \mathrm{H}, \mathrm{ArH}), 7.82\left(\mathrm{~d}, J=7.8 \mathrm{~Hz}, 1 \mathrm{H}, \mathrm{C}_{3} \mathrm{H}\right), 8.85(\mathrm{~d}, J=7.8$ $\left.\mathrm{Hz}, 1 \mathrm{H}, \mathrm{C}_{1} \mathrm{H}\right), 9.81$ (s, $\left.1 \mathrm{H}, \mathrm{NH}\right)$; MS: $m / z(\%): 489(\mathrm{M}+4,10)$, 487 (M+2, 40), 485 (M+, 70), 450 (30), 415 (20), 361 (50), 325 (100), 168 (90), 125 (75). Anal. Calcd. for $\mathrm{C}_{23} \mathrm{H}_{17} \mathrm{~N}_{3} \mathrm{OS}_{2} \mathrm{Cl}_{2}$ (486.45): C, 56.79; H, 3.52; N, 8.64. Found: C, 56.73; H, 3.55; $\mathrm{N}, 8.67$.

2-(4-Chloro-12-methyl-16,17-dihydro-15-thia-6,11diazacyclopenta $[a]$ phenanthren-7-ylsulfanyl)-N-(2,4dichlorophenyl)acetamide (3e): Yield $(0.468 \mathrm{~g}, 90 \%)$; $\mathrm{R}_{\mathrm{f}}$ (toluene/ethyl acetate 8:2) 0.71, m.p.: $229^{\circ} \mathrm{C}$; IR (KBr, $v_{\max }$, $\left.\mathrm{cm}^{-1}\right)$ : $3244(\mathrm{NH}), 2920,2850,1683(\mathrm{C}=\mathrm{O}), 1546,1514,1442$, $1305,1168,954 ;{ }^{1} \mathrm{H}$ NMR $\left(\mathrm{CDCl}_{3}\right): \delta 2.78\left(\mathrm{~s}, 3 \mathrm{H}, \mathrm{CH}_{3}\right), 3.57$ $\left(\mathrm{t}, J=6.9 \mathrm{~Hz}, 2 \mathrm{H}, \mathrm{CH}_{2} \mathrm{CH}_{2} \mathrm{~S}\right), 3.64(\mathrm{t}, J=6.9 \mathrm{~Hz}, 2 \mathrm{H}$, $\mathrm{CH}_{2} \mathrm{CH}_{2} \mathrm{~S}$ ), 4.49 (s, $2 \mathrm{H}, \mathrm{CH}_{2}$ ), 7.23-7.27 (m, 2H, $\left.\mathrm{ArH}\right), 7.56$ $\left(\mathrm{t}, J=8.1 \mathrm{~Hz}, 1 \mathrm{H}, \mathrm{C}_{2} \mathrm{H}\right), 7.84\left(\mathrm{~d}, J=8.1 \mathrm{~Hz}, 1 \mathrm{H}, \mathrm{C}_{3} \mathrm{H}\right), 8.30$ $(\mathrm{d}, J=7.9 \mathrm{~Hz}, 1 \mathrm{H}, \mathrm{ArH}), 9.01\left(\mathrm{~d}, J=8.1 \mathrm{~Hz}, 1 \mathrm{H}, \mathrm{C}_{1} \mathrm{H}\right), 9.81$ (s, 1H, NH); MS: $\mathrm{m} / \mathrm{z}$ (\%): $526(\mathrm{M}+6,20), 524(\mathrm{M}+4,30)$, $522(\mathrm{M}+2,50), 520(\mathrm{M}+, 90), 368(40), 367$ (100), $325(20)$, 135 (20), 116 (15); Anal. Calcd. for $\mathrm{C}_{23} \mathrm{H}_{16} \mathrm{~N}_{3} \mathrm{OS}_{2} \mathrm{Cl}_{3}(520.89)$ : C, 53.04; H, 3.10; N, 8.07. Found: C, 53.07; H, 3.06; N, 8.10.

N-(2-Chloro-6-fluorophenyl)-2-(4-chloro-12-methyl16,17-dihydro-15-thia-6,11-diazacyclopenta $[a]$ phenanthren-7-ylsulfanyl)acetamide (3f): Yield (0.438 g, $87 \%)$; m.p.: $263{ }^{\circ} \mathrm{C}$; IR $\left(\mathrm{KBr}, v_{\max }, \mathrm{cm}^{-1}\right)$ : $3250(\mathrm{NH}), 3041,2922$, $1685(\mathrm{C}=\mathrm{O}), 1546,1444,1280,1168,952 ;{ }^{1} \mathrm{H} \mathrm{NMR}\left(\mathrm{CDCl}_{3}\right)$ : $\delta 2.74\left(\mathrm{~s}, 3 \mathrm{H}, \mathrm{CH}_{3}\right), 3.51$ (t, $\left.J=6.7 \mathrm{~Hz}, 2 \mathrm{H}, \mathrm{CH}_{2} \mathrm{CH}_{2} \mathrm{~S}\right), 3.59$ $\left(\mathrm{t}, J=6.7 \mathrm{~Hz}, 2 \mathrm{H}, \mathrm{CH}_{2} \mathrm{CH}_{2} \mathrm{~S}\right), 4.51\left(\mathrm{~s}, 2 \mathrm{H}, \mathrm{CH}_{2}\right), 7.24-7.32$ (m, 4H, ArH), $7.55\left(\mathrm{t}, J=8.0 \mathrm{~Hz}, 1 \mathrm{H}, \mathrm{C}_{2} \mathrm{H}\right), 7.91(\mathrm{~d}, J=8.0$ $\left.\mathrm{Hz}, 1 \mathrm{H}, \mathrm{C}_{3} \mathrm{H}\right), 8.85\left(\mathrm{~d}, J=8.0 \mathrm{~Hz}, 1 \mathrm{H}, \mathrm{C}_{1} \mathrm{H}\right), 10.06(\mathrm{~s}, 1 \mathrm{H}$, $\mathrm{NH})$; MS: $m / z(\%): 507(\mathrm{M}+4,10), 505(\mathrm{M}+2,30), 503(\mathrm{M}+$, 100), 436 (40), 393 (20), 361 (40), 319 (30), 196 (80), 142 (50). Anal. Calcd. for $\mathrm{C}_{23} \mathrm{H}_{16} \mathrm{~N}_{3} \mathrm{OS}_{2} \mathrm{Cl}_{2} \mathrm{~F}(504.44)$ : C, 54.77; H, $3.20 ; \mathrm{N}, 8.33$. Found: $\mathrm{C}, 54.74 ; \mathrm{H}, 3.23 ; \mathrm{N}, 8.36$.

2-(4-Chloro-12-methyl-16,17-dihydro-15-thia-6,11diazacyclopenta $[a]$ phenanthren-7-ylsulfanyl)-N-(3-chloro4-trifluoromethylphenyl)acetamide (3g): Yield ( $0.448 \mathrm{~g}, 81$ \%); m.p.: $243^{\circ} \mathrm{C}$; IR (KBr, $\left.v_{\max }, \mathrm{cm}^{-1}\right): 3317$ (NH), 2922, 1691 $(\mathrm{C}=\mathrm{O}), 1545,1483,1223,1130,950 ;{ }^{1} \mathrm{H} \mathrm{NMR}\left(\mathrm{CDCl}_{3}\right): \delta$ 2.70 (s, $3 \mathrm{H}, \mathrm{CH}_{3}$ ), 3.50 (t, $J=7.2 \mathrm{~Hz}, 2 \mathrm{H}, \mathrm{CH}_{2} \mathrm{CH}_{2} \mathrm{~S}$ ), 3.57 (t, $\left.J=7.2 \mathrm{~Hz}, 2 \mathrm{H}, \mathrm{CH}_{2} \mathrm{CH}_{2} \mathrm{~S}\right), 4.29\left(\mathrm{~s}, 2 \mathrm{H}, \mathrm{CH}_{2}\right), 7.34(\mathrm{~d}, J=8.4$ 
$\mathrm{Hz}, 1 \mathrm{H}, \mathrm{ArH})$, 7.53-7.63 (m, 2H, ArH, $\left.\mathrm{C}_{2} \mathrm{H}\right), 7.87-7.89$ (m, $\left.2 \mathrm{H}, \mathrm{ArH}, \mathrm{C}_{3} \mathrm{H}\right), 9.03\left(\mathrm{~d}, J=8.4 \mathrm{~Hz}, 1 \mathrm{H}, \mathrm{C}_{1} \mathrm{H}\right), 10.21(\mathrm{~s}, 1 \mathrm{H}$, $\mathrm{NH}$ ); Anal. Calcd. for $\mathrm{C}_{24} \mathrm{H}_{16} \mathrm{~N}_{3} \mathrm{OS}_{2} \mathrm{Cl}_{2} \mathrm{~F}_{3}$ (554.44): C, 51.99; H, 2.91; N, 7.58. Found: C, 52.03; H, 2.90; N, 7.55.

N-(2,4-bis-trifluoromethylphenyl)-2-(4-chloro-12methyl-16,17-dihydro-15-thia-6,11-diazacyclopenta[a]phenanthren-7-ylsulfanyl)acetamide (3h): Yield $(0.531 \mathrm{~g}$, $91 \%)$; m.p.: $150{ }^{\circ} \mathrm{C}$; IR (KBr, $\left.v_{\max }, \mathrm{cm}^{-1}\right)$ : $3244(\mathrm{NH}), 2918$, 2850, $1678(\mathrm{C}=\mathrm{O}), 1546,1433,1142,1042,954 ;{ }^{1} \mathrm{H}$ NMR $\left(\mathrm{CDCl}_{3}\right): \delta 2.72\left(\mathrm{~s}, 3 \mathrm{H}, \mathrm{CH}_{3}\right), 3.50(\mathrm{t}, J=6.5 \mathrm{~Hz}, 2 \mathrm{H}$, $\mathrm{CH}_{2} \mathrm{CH}_{2} \mathrm{~S}$ ), 3.60 (t, $J=6.5 \mathrm{~Hz}, 2 \mathrm{H}, \mathrm{CH}_{2} \mathrm{CH}_{2} \mathrm{~S}$ ), 4.43 (s, $2 \mathrm{H}$, $\left.\mathrm{CH}_{2}\right), 7.42-7.51\left(\mathrm{~m}, 2 \mathrm{H}, \mathrm{ArH}, \mathrm{C}_{2} \mathrm{H}\right), 7.60(\mathrm{~d}, J=8.1 \mathrm{~Hz}, 1 \mathrm{H}$, $\left.\mathrm{C}_{3} \mathrm{H}\right), 7.74(\mathrm{~d}, J=6.9 \mathrm{~Hz}, 1 \mathrm{H}, \mathrm{ArH}), 8.42(\mathrm{~s}, 1 \mathrm{H}, \mathrm{ArH}), 8.98$ $\left(\mathrm{d}, J=8.1 \mathrm{~Hz}, 1 \mathrm{H}, \mathrm{C}_{1} \mathrm{H}\right), 9.64(\mathrm{~s}, 1 \mathrm{H}, \mathrm{NH})$; Anal. Calcd. for $\mathrm{C}_{26} \mathrm{H}_{19} \mathrm{~N}_{3} \mathrm{OS}_{2} \mathrm{ClF}_{5}(584.03): \mathrm{C}, 51.70 ; \mathrm{H}, 3.34 ; \mathrm{N}, 6.96$. Found: C, 51.73; H, 3.30; N, 6.94 .

Antibacterial studies: Eight bacterial species such as Escherichia coli, Staphylococcus aureus, Bacillus subtilis, Bacillus cereus, Proteus valgaris, Streptococcus sps, Bacillus megaterium and Pseudomonas aeroginosa were tested for antimicrobial activiy by agar well diffusion method. Nutrient agar (tryptone $1 \%$, yeast extract $0.5 \%, \mathrm{NaCl} 0.5 \%$, agar 2.5 $\%, 1000 \mathrm{~mL}$ of distilled water, PH 7.0) which was autoclaved under $121{ }^{\circ} \mathrm{C}$ for at least 20 min was inovculated by spread plate technique with bacteria from $24 \mathrm{~h}$ old culture having approximately $10^{4}-10^{6} \mathrm{CFU}$ (colony forming unit) per $\mathrm{mL}$. After inoculation wells were made by sterile metallic boarer and the compound to be tested were loaded $(100 \mu \mathrm{L})$ in the well. The plates were incubated for $24 \mathrm{~h}$ and the activity was determined by measuring zone of inhibition around the well. All compounds was prepared in N,N-dimethyl formamide
(DMF), DMF was loaded as control. The average value of inhibition zone was calculated. All the experiment was carried out in triplicate for each bacterial culture. Ampicilin and streptomycin were used as antibacterial standard drugs. Zone of inhibition were determined for compounds $\mathbf{2}$ to $\mathbf{3}(\mathbf{a}-\mathbf{h})$ the results are mentioned in Table-1.

\section{RESULTS AND DISCUSSION}

We already reported the synthesis of methylbenzo-[h][1,6]naphthyridines (1) by using 4-aminoquinolines and $\alpha$-acetyl $\gamma$-butyrolactone [15]. The iminechloride moiety was converted into thiol by refluxing compound $\mathbf{1}$ with thiourea in acetic acid furnished 4-chloro-12-methyl-16,17-dihydro-15-thia6,11-diazza-cyclopenta[ $a]$ phenanthrene-7-thiol (2) in $90 \%$ yield $[22,23]$. The structure of compound $\mathbf{2}$ showed thiol $(\mathrm{SH})$ stretching at $3350, \mathrm{NH}$ at $3330 \mathrm{~cm}^{-1}$ and $(\mathrm{C}=\mathrm{S})$ at $1660 \mathrm{~cm}^{-1}$ in its IR spectra. It was observed that alkylation reaction on compound 2 with bromoester/substituted $N$-phenyl bromoacetamide in presence of anhydrous $\mathrm{K}_{2} \mathrm{CO}_{3}$ in DMF at room temperature gave only $S$-alkylated compound $\mathbf{3}$ in $82-91 \%$ yield, which showed absence of $(\mathrm{C}=\mathrm{S})$ stretching frequency indicates the $S$-alkylation in 3. The ${ }^{1} \mathrm{H}$ NMR spectrum in $\mathrm{CDCl}_{3}$ of $\mathbf{3 a}$ showed triplets at $\delta 3.45$ and $\delta 3.56 \mathrm{ppm}$ with $J=8.1 \mathrm{~Hz}$ were designate for $\mathrm{CH}_{2} \mathrm{CH}_{2} \mathrm{~S}$. The up field triplet at $\delta 1.28 \mathrm{ppm}$ and down field quartet at $\delta 4.25 \mathrm{ppm}$ with $J=7.2 \mathrm{~Hz}$ were designate for $\mathrm{OCH}_{2} \mathrm{CH}_{3}$. The peak at $\delta 4.29 \mathrm{ppm}$ was assigned for methylene protons, the other aromatic protons were resonance at expected splitting pattern and chemical shifts. The EIMS of compound 3a showed $\mathrm{M}+$ at 405 and $\mathrm{M}+2$ at $407 \mathrm{~m} / z$, corresponds to chlorine atom (Scheme-I).

TABLE-1

ANTIMICROBIAL ACTIVITY OF SYNTHESIZED COMPOUNDS TESTED AGAINST BACTERIAL SPECIES

\begin{tabular}{|c|c|c|c|c|c|c|c|c|}
\hline \multirow{2}{*}{ Compd. No. } & \multicolumn{8}{|c|}{ Antibacterial inhibition zone in diameter $(\mathrm{mm})$} \\
\hline & S. aureus & E. coli & B. subtilis & P. aeroginosa & P. valgaris & B. cereus & Streptococcus $s p$ & B. megaterium \\
\hline 2 & - & - & - & - & - & - & - & - \\
\hline 3a & - & - & - & - & - & - & - & - \\
\hline $\mathbf{3 b}$ & - & - & - & - & - & - & - & - \\
\hline $3 c$ & - & - & - & - & - & - & - & - \\
\hline 3d & - & 14 & - & - & - & - & - & - \\
\hline $3 e$ & - & - & - & - & - & - & - & - \\
\hline $3 f$ & - & 08 & - & - & - & - & - & 11 \\
\hline $3 g$ & - & - & - & - & - & - & - & - \\
\hline $3 h$ & - & 08 & - & - & - & - & - & 08 \\
\hline *Ampicillin & 45 & 35 & 40 & 45 & 34 & 33 & 40 & 15 \\
\hline *Streptomycin & 13 & 00 & 15 & 00 & 34 & 40 & 00 & 00 \\
\hline
\end{tabular}

*Ampicillin and streptomycin are used as standard antibacterial agents.<smiles>Cc1nc2c(c(Cl)nc3c(Cl)cccc32)c(Cl)c1CCCl</smiles>

1

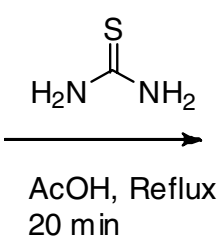

$20 \mathrm{~min}$<smiles>Cc1nc2c(c(S)nc3c(Cl)cccc32)c2c1CCS2</smiles>

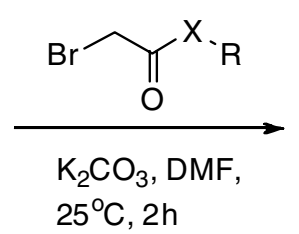

$25^{\circ} \mathrm{C}, 2 \mathrm{~h}$<smiles>[R][X]C(=O)CSc1nc2c(Cl)cccc2c2nc(C)c3c(c12)SCC3</smiles>

3(a-h) $(82-91 \%)$

3a: $\mathrm{X}=\mathrm{O}, \mathrm{R}=\mathrm{C}_{2} \mathrm{H}_{5} ; 3 \mathbf{b}: \mathrm{X}=\mathrm{NH}, \mathrm{R}=4-\mathrm{MeC}_{6} \mathrm{H}_{4} ; \mathbf{3 c}: \mathrm{X}=\mathrm{NH}, \mathrm{R}=4-\mathrm{FC}_{6} \mathrm{H}_{4} ; 3 \mathbf{d}: \mathrm{X}=\mathrm{NH}, \mathrm{R}=4-\mathrm{ClC}_{6} \mathrm{H}_{4}$; 3e: $X=N H, R=2,4-\mathrm{ClC}_{6} \mathrm{H}_{3} ;$ 3f: $X=\mathrm{NH}, \mathrm{R}=2-\mathrm{Cl}, 6-\mathrm{FC}_{6} \mathrm{H}_{3} ; \mathbf{3 g}: \mathrm{X}=\mathrm{NH}, \mathrm{R}=3-\mathrm{Cl}, 4-\mathrm{CF}_{3} \mathrm{C}_{6} \mathrm{H}_{3} ; \mathbf{3 h}: \mathrm{X}=\mathrm{NH}, \mathrm{R}=2,4-\mathrm{CF}_{3} \mathrm{C}_{6} \mathrm{H}_{3}$ 
Antimicrobial activities: To detect antimicrobial activities of compounds were screened against cultures of eight bacterial species, namely, Staphylococcus aureus, Escherichia coli, Bacillus subtilis, Pseudomonas aeroginosa, Proteus valgaris, Bacillus cereus, Streptococcus sp and Bacillus megaterium by agar well diffusion technique. Tested compounds showed moderate activity against the bacterial strains was mentioned in Table-1. Inhibition zone diameter (IZD in $\mathrm{mm}$ ) was measured with solution concentration of each compound was $1.0 \mathrm{mg} / \mathrm{mL}$. Streptomycin and ampicillin were used as an reference antibiotics antibacterial agents to evaluate the potency of tested compounds. The inhibitory concentration (MIC) of the biologically active compounds was determined by using two-fold dilution method. The results of newly synthesized compounds were obtained are shown in Table- 1 . The cyclopenta $[a]$ phenanthren 3(a-h) derivatives were showed antibacterial activity against $E$. coli and B. megaterium species.

\section{Conclusion}

The convenient synthesis of benzo[ $h][1,6]$ naphthyridines and cyclopenta $[a]$ phenanthrens as a useful tool in the development of iminoether and iminothioether derivatives was developed. The antimicrobial activity study revealed that compounds $\mathbf{3 d}, \mathbf{3 f}$ and $\mathbf{3 h}$ showed moderate antimicrobial activity.

\section{ACKNOWLEDGEMENTS}

The author thankful to Board of College and University Development (BCUD) Savitribai Phule Pune University and UGC New Delhi, India for financial support to carry out this research work. The author also thanks to Savitribai Phule Pune University, Pune, IIT-Powai Mumbai and K.T.H.M. College Nashik, for spectral and analytical facilities.

\section{CONFLICT OF INTEREST}

The authors declare that there is no conflict of interests regarding the publication of this article.

\section{REFERENCES}

1. A.K. Munusamy, M.S. Kumar, P.D. Subramanian and P. Rajendran, $J$. Chin. Chem. Soc., 64, 138 (2017); https://doi.org/10.1002/jccs.201600196.

2. K. Ramkumar, E. Serrao, S. Odde and N. Neamati, Med. Res. Rev., 30, 890 (2010); https://doi.org/10.1002/med.20194.

3. B.A. Johns, J.G. Weatherhead, S.H. Allen, J.B. Thompson, E.P. Garvey, S.A. Foster, J.L. Jeffrey and W.H. Miller, Bioorg. Med. Chem. Lett., 19, 1807 (2009); https://doi.org/10.1016/j.bmcl.2009.01.089.

4. J.Y. Melamed, M.S. Egbertson, S. Varga, J.P. Vacca, G. Moyer, L. Gabryelski, P.J. Felock, K.A. Stillmock, M.V. Witmer, W. Schleif, D.J. Hazuda, Y. Leonard, L. Jin, J.D. Ellis and S.D. Young, Bioorg. Med. Chem. Lett., 18, 5307 (2008); https://doi.org/10.1016/j.bmcl.2008.08.038
5. M.S. Egbertson, H.M. Moritz, J.Y. Melamed, W. Han, D.S. Perlow, M.S. Kuo, M. Embrey, J.P. Vacca, M.M. Zrada, A.R. Cortes, A. Wallace, Y. Leonard, D.J. Hazuda, M.D. Miller, P.J. Felock, K.A. Stillmock, M.V. Witmer, W. Schleif, L.J. Gabryelski, G. Moyer, J.D. Ellis, L. Jin, W. Xu, M.P. Braun, K. Kassahun, N.N. Tsou and S.D. Young, Bioorg. Med. Chem. Lett., 17, 1392 (2007); https://doi.org/10.1016/j.bmcl.2006.11.080.

6. G. Falardeau, H. Lachance, A. St-Pierre, C.G. Yannopoulos, M. Drouin, J. Bédard and L. Chan, Bioorg. Med. Chem. Lett., 15, 1693 (2005); https://doi.org/10.1016/j.bmcl.2005.01.050.

7. L. Chan, H. Jin, T. Stefanac, J.F. Lavallee, G. Falardeau, W. Wang, J. Bedard, S. May and L. Yuen, J. Med. Chem., 42, 3023 (1999); https://doi.org/10.1021/jm9902483.

8. A.M. Thompson, C.J.C. Connolly, J.M. Hamby, S. Boushelle, B.G. Hartl, A.M. Amar, A.J. Kraker, D.L. Driscoll, S.J. Steinkampf, S.J. Patmore, P.W. Vincent, B.J. Roberts, W.L. Elliott, W. Klohs, W.R. Leopold, H.D.H. Showalter and W.A. Denny, J. Med. Chem., 43, 4200 (2000); https://doi.org/10.1021/jm000161d.

9. S. Vanlaer, A. Voet, C. Gielens, M. De Maeyer and F. Compernolle, Eur. J. Org. Chem., 643 (2009); https://doi.org/10.1002/ejoc.200800972.

10. (a) E.-S. Badawey and T. Kappe, J. Heterocycl. Chem., 32, 1003 (1995); https://doi.org/10.1002/jhet.5570320355.

(b) E.-S. Badawey, J. Heterocycl. Chem., 32, 229 (1996); https://doi.org/10.1002/jhet.5570330202.

11. T. Ichikawa, J.C. Lamb, P.I. Christensson, B. Hartly-Asp and J.T. Issacs, Cancer Res., 52, 3022 (1992)

12. S.C. Kuo, H.Z. Lee, J.P. Juang, Y.T. Lin, T.S. Wu, D. Lednicer, J.J. Chang, K.D. Paull and C.M. Lin, J. Med. Chem., 36, 1146 (1993); https://doi.org/10.1021/jm00061a005.

13. Q. Liu, J.W. Chang, J. Wang, S.A. Kang, C.C. Thoreen, A. Markhard, W. Hur, J. Zhang, T. Sim, D.M. Sabatini and N.S. Gray, J. Med. Chem., 53, $7146(2010)$; https://doi.org/10.1021/jm101144f

14. Q. Liu, J. Wang, S.A. Kang, C.C. Thoreen, T. Ahmed, D.M. Sabatini, W. Hur and N.S. Gray, J. Med. Chem., 54, 1473 (2011); https://doi.org/10.1021/jm101520v.

15. R.B. Toche, B.P. Pagar, R.R. Zoman, G.B. Shinde and M.N. Jachak, Tetrahedron, 66, 5204 (2010); https://doi.org/10.1016/j.tet.2010.04.085.

16. R.B. Toche, B.K. Ghotekar, M.A. Kazi and M.N. Jachak, Monatsh. Chem., 140, 235 (2009); https://doi.org/10.1007/s00706-008-0062-x.

17. R.B. Toche, B.K. Ghotekar, D.B. Kendre, M.A. Kazi and M.N. Jachak, J. Heterocycl. Chem., 45, 1711 (2008); https://doi.org/10.1002/jhet.5570450624.

18. R.B. Toche, B.K. Ghotekar, M.A. Kazi, S.P. Patil and M.N. Jachak, Scholarly Res. Exch., Article ID 434329 (2008); https://doi.org/10.3814/2008/434329.

19. B.K. Ghotekar, M.A. Kazi, M.N. Jachak and R.B. Toche, Can. J. Chem., 86, 1070 (2008); https://doi.org/10.1139/v08-155.

20. R.B. Toche, B.K. Ghotekar, M.A. Kazi, D.B. Kendre and M.N. Jachak, Tetrahedron, 63, 8157 (2007) https://doi.org/10.1016/j.tet.2007.05.123.

21. R.V. Rote, D.P. Shelar, S.R. Patil, S.S. Shinde, R.B. Toche and M.N. Jachak, J. Fluoresc., 21, 453 (2011); https://doi.org/10.1007/s10895-010-0704-3.

22. E.-S. Badawey and T. Kappe, Eur. J. Med. Chem., 32, 815 (1997); https://doi.org/10.1016/S0223-5234(99)80067-1.

23. E.-S.A.M. Badawey, J. Heterocycl. Chem., 33, 229 (1996); https://doi.org/10.1002/ihet.5570330202. 